\title{
MOTOR PERFORMANCE IN PATIENTS WITH KNEE OSTEOARTHRITIS AFTER 8-WEEK HOME EXERCISE PROGRAM
}

\author{
Jelena SokK ${ }^{1}$, Monika Rätsepsoo ${ }^{1,3}$, Tatjana Kums ${ }^{1}$, \\ Jaan Ereline ${ }^{1}$, Tiit Haviko ${ }^{2}$, Helena Gapeyeva ${ }^{1}$, Mati PäÄsuke ${ }^{1}$ \\ ${ }^{1}$ Institute of Sport Sciences and Physiotherapy, University of Tartu, Tartu, Estonia \\ ${ }^{2}$ Department of Orthopaedics and Traumatology, Tartu University Hospital, Tartu, \\ Estonia \\ ${ }^{3}$ Doctoral School of Behavioural, Social and Health Sciences, University of Tartu, \\ Tartu, Estonia
}

\begin{abstract}
On patients with knee osteoarthritis (OA) the muscle strength, gait and everyday activities performance capacity decreased, though patients care started many years before. Patients care includes supervised physiotherapy sessions and home exercise performing. Research goal was to assess the improvement in patients with knee OA motor performance with home exercise program (HEP) 8-weeks before total knee arthroplasty (TKA). Hypothesis: 8-week HEP improve legs motor performance in patients with knee OA. Ten female patients with knee OA with the mean $( \pm \mathrm{SE})$ age of $62.7 \pm 2.3 \mathrm{yrs}$; ten age- and gender-matched healthy controls with the mean $( \pm \mathrm{SE})$ age of $62.6 \pm 1.1$ yrs. Motor performance was assessed by isometric strength of the quadriceps femoris (QF) and hamstring (HM) muscles, gait, Five-Time-Sit-to-Stand (FTSTS) test and knee active range of motion (aROM) before and after 8-week HEP. Outcome was measured by Western Ontario and McMaster Universities Osteoarthritis Index (WOMAC). After 8-week HEP, QF and HM isometric strength, knee aROM during flexion and the gait stride length for the involved leg increased $(\mathrm{p}<0.05)$ compared to pre-exercising level. The WOMAC score increased and FTSTS test time shortened $(\mathrm{p}<0.05)$ after 8 -week HEP. Difference in measured parameters between the patients involved leg decreased as compared to uninvolved leg and controls. Study showed that after 8-week HEP improved involved leg thigh's muscle strength, knee aROM, and stride length. Sit to stand test performance and patient's
\end{abstract}


self-assessed condition improved. In conclusion, HEP with at least 8 weeks should be recommended for patients with knee OA before TKA.

Keywords: knee osteoarthritis; QF muscle isometric strength (MVC) force; HM muscle MVC force; gait; home exercise program

\section{INTRODUCTION}

Knee osteoarthritis (OA) is a chronic health problem, affecting adults 50 yrs of age or older [34] and leading to decreasing mobility [20], deconditioning, reduced functional status and quality of life $[15,19]$ in patients. It has been found that in patients with knee OA muscle strength and muscle power decreases with age. Low muscle strength and power is associated with patients' poor mobility [17] and decreased knee range of motion (ROM) affect patients' ambulation function [32]. According to this the gait parameters are affected in patients with knee OA before and after total knee arthroplasty (TKA) [13].

It has been advised to use muscle strengthening program [7] and in functional exercises to relieve pain in patients with knee OA [22]. Education, exercising and weight loss are effective in the long term as first-line treatment [26]. Therapeutic exercises and physical activity are important for patients with knee OA in any stage of the disease [23].

The study of Hurley and Scott [14] demonstrated that the increase in quadriceps femoris $(\mathrm{QF})$ muscle strength associated with disability reduction in patients with knee OA after 12 weeks of outpatients' physiotherapy sessions twice a week. The increase in QF muscle strength and motor function after 4-6-week pre-rehabilitation in patients with severe knee OA before TKA has been shown in several studies $[17,18,25]$ where patients with knee OA done therapeutic exercises under physiotherapist supervision in the clinic. In patients with medial knee OA, the 8-week training of hip abductors within home exercising program improved the function of the knee and reduced pain [30]. On the other hand, the study of Rodgers et al. [24] that assessed the 6-week pre-operative physiotherapy effect, failed to support the routine use of pre-operative physiotherapy for patients before TKA. Ackerman and Bennell [1] concluded that pre-operative physiotherapy programs are not effective in improving the outcome after TKA. Patients who participated in an exercise intervention program three times per week, for 8 weeks before TKA, had a better quality of life 3 months after TKA [4].

The results yielded by pre-operative rehabilitation studies are controversial and the respective research has concentrated on knee ROM, QF strength 
and leg extensors strength, and also on subjective questionnaires in patients with knee OA.

To our best knowledge, no studies have been conducted on the effect of home exercise program in patients with knee OA 8-week before TKA. The purpose of this study was to measure the changes in motor function in patients with knee OA after 8-week home exercise program (HEP). It was hypothesised that after 8-week HEP in patients with knee OA motor performance of the legs improve.

\section{MATERIAL AND METHODS}

\section{Subjects}

Ten female subjects with knee OA (HEP group) aged 59 to 74 years waiting for unilateral TKA in stage III-IV according to the Kellgren-Lawrence Scale [16] participated in this study. The subjects were recruited by orthopaedic surgeons in the Department of Orthopaedics and Traumatology of Tartu University Hospital, between February and December 2011. The mean \pm SE age of the subjects in the HEP group was $62.8 \pm 2.3 \mathrm{yrs}$, height $160.5 \pm 1.8 \mathrm{~cm}$, body mass $82.4 \pm 4.4 \mathrm{~kg}$, and BMI $31.5 \pm 1.5 \mathrm{~kg} / \mathrm{m}^{2}$. The data of HEP group subjects were compared to ten healthy subjects as controls (mean $\pm \mathrm{SE}$ age of $62.1 \pm 1.8 \mathrm{yrs}$, height $161.0 \pm 1.8 \mathrm{~cm}$, body mass $70.6 \pm 4.1 \mathrm{~kg}$, and BMI $\left.27.2 \pm 1.5 \mathrm{~kg} / \mathrm{m}^{2}\right)$.

The exclusion criteria for HEP group subjects was inflammatory arthritis, obesity (BMI $\left.>35 \mathrm{~kg} / \mathrm{m}^{2}\right)$, implanted pacemakers, progressive neurological disease, diabetes, head injuries, cardiac diseases NYHA III-IV, osteoporosis, deep vein thrombosis, COPD, Parkinson disease. Healthy subjects (control group) exclusion criteria were joint replacement of any joint of lower extremities, painful joints, or any other criteria listed for the subjects in HEP group.

The subjects were instructed and the thigh muscle strength and gait testing procedures were demonstrated $24-48$ hours before collecting the first data. This was followed by a practical session to familiarise the subjects with procedures. Before testing, each subject underwent a 10-min warm-up consisting of ride by bicycle and stretching exercises. Both legs were tested, the uninvolved leg was tested first. The data collection was done before and after 8-week HEP, whereas the control subjects evaluated once. Baseline and follow-up study evaluations were the following: isometric strength of the $\mathrm{QF}$ and hamstring (HM) muscles, gait assessment, FTSTS test, WOMAC questionnaire and knee aROM. 
Before participation in the study, all subjects gave their written informed consent. The study carried the approval of the University of Tartu Ethics Committee.

\section{Home exercise program}

The pre-operative therapeutic 8-week HEP was mainly oriented to muscle strengthening exercises with elastic band (Thera-Band, System of Progressive Exercise, US), in addition, the subjects did stretching, balance and walking exercises. At the first meeting, the physiotherapist gave a detailed verbal, visual and hands-on instruction on the exercise program to the HEP group subjects, provided them with the elastic band set, printout of the exercise program with pictures and explanations, and diary. The subjects recorded daily for how many times they did each exercise, the training time, perception of pain before and after HEP, time spent in outdoor walking and the how many stairs they had walked during the day. All exercise done with both legs. Pain before and after HEP was assessed using Borg Scale of pain.

\section{Measurement of isometric maximal voluntary contraction force}

MVC force of the QF and HM muscles was measured by hand-held dynamometer (Lafayette Manual Muscle Test System). During QF muscle MVC force testing the subject was seated on the investigation chair with knee and hip joints flexed to $90 \mathrm{deg}$, the subject body was fixed with a special belt. Hand-held dynamometer was placed proximal to the ankle on anterior surface of leg with special belt. During HM muscle MVC force testing, the subject was in the prone position on the investigation table; knee was flexed $30 \mathrm{deg}$ with special pillow. Hand-held dynamometer was placed proximal to the ankle on posterior surface of leg with a special belt [29]. Belt was fixated to standardized attachments on the investigation chair for extension and on the investigation table for flexion. The length of the belt allowed for an isometric contraction to be performed with the knee at $90^{\circ}$ during flexion and $30^{\circ}$ during extension. The positions were supervised by the investigator and the subjects were encouraged to act in the showed way. The subjects were required to exert isometric strength pushing against the dynamometer for approximately $3 \mathrm{~s}$ during each contraction, and instructed to "push as hard as possible". The best results from 3 attempts were taken as MVC force, whereas parameters were normalized with the subject's body mass (kg). A rest period of 1 min was allowed between attempts. Stark et al. [31] in systematic review found minimal differences between hand-held dynamometry and isokinetic testing. 


\section{Measurement of gait}

Gait kinematic temporal and distance characteristics were recorded using optic-electronic movement analysis system ELITE (BTS, Italy) with six infrared cameras with the sampling rate of $100 \mathrm{~Hz}$, and the ground reaction forces (GRF) were measured simultaneously with two Kistler platforms (Kistler 9286A, Sweden). Twenty passive reflecting spherical markers with the diameter of $15 \mathrm{~mm}$ were attached to the selected anatomical points of body according to the Davis [9] biomechanical model. Gait kinematic temporal and distance characteristics data and GRF were computed by and ELITE Clinic software (BTS SpA, Italy). Values of temporal parameters [stance time (s), stride time (s)) and distance parameters (step length (m), velocity $(\mathrm{m} / \mathrm{s})$ and stride length $(\mathrm{m})]$ were used for future analysis, whereas step length and velocity parameters were normalized with the subject's leg length (m). The subjects were requested to walk at the comfortable velocity along a $5.3 \mathrm{~m}$ walkway. Three to five trials were recorded and the trial with stable walking cadence (without de- or accelerations) was taken for analysis.

Gait analysis is a reproducible and objective method of quantifying changes in gait before and after treatment in patients with different conditions, including knee OA [33].

\section{Measurement of sit to stand performance}

Five-Time-Sit-to-Stand (FTSTS) test has been used for measuring lower extremity function [8], it measures the time required to rise from a chair and sit down for 5 repetitions. The subject was seated on an armless chair with knees at $90^{\circ}$ flexion and hold arms across the chest. At the beginning and end of the test the subject was in the seated position. The time (measured with a hand-held stop watch) was recorded during which the subject transferred as quickly as possible for 5 times between the sitting and standing position. The subjects were instructed to stand up fully on each repetition. When the subject felt the pain, or did not stand fully up the test was interrupted. Two trials were recorded and the fastest successful one was used for analysis. FTSTS test conducted same person.

\section{Knee flexion range of motion measurement}

Knee aROM during flexion was measured in prone position with the standard long arm goniometer. The axis of the goniometer was placed over lateral femoral epicondyle, the proximal end of the goniometer was aligned with the great trochanter of the femur and the distal arm was aligned with the lateral malleolus. The subjects were instructed to maximally flex the knee and draw 
the heel toward the buttocks. The maximal knee flexion was recorded from three trials. Goniometric knee flexion measurements are highly reliable in persons with knee OA [12].

\section{Patients' self-assessment of knee joint function}

For the assessment of the subject's condition the WOMAC was used. The WOMAC measures five items for pain (score range 0-20), two for stiffness (score range $0-8$ ), and 17 for functional limitation (score range 0-68). Higher WOMAC scores indicate worse pain, stiffness, and functional limitations. The subjects completed the questionnaires independently. Studies showed that WOMAC score is valid instrument for assessing patients with knee and hip OA [2].

\section{Statistical analysis}

Data are expressed as mean and standard error $( \pm$ SE). One-way analysis of variance (ANOVA) followed by Tukey a post hoc comparison was used to evaluate differences between the groups and between the involved and uninvolved leg in subjects with knee OA. A paired t-test was used to evaluate differences between pre- and post-HEP session characteristics. A level of $\mathrm{p}<0.05$ was selected to indicate statistical significance.

\section{RESULTS}

Before 8-week HEP, subjects with knee OA showed a significant reduction $(\mathrm{p}<0.05)$ in the QF and HM muscle MVC force and knee aROM during flexion for the involved leg as compared with the uninvolved leg and controls (Table 1). For the involved and uninvolved leg the stance and stride time (Table 1$)$ were longer $(\mathrm{p}<0.05)$, whereas the step and stride length was shorter, and velocity was lower $(\mathrm{p}<0.05)$ as compared with controls.

After 8-week HEP, the QF muscle MVC force and knee aROM during flexion increased $(\mathrm{p}<0.05)$ for the subjects' involved leg, whereas the knee aROM remained significantly lower $(\mathrm{p}<0.05)$ as compared with the uninvolved knee and controls. The HM muscle MVC force remained lower $(\mathrm{p}<0.05)$ for both legs as compared with controls, whereas the HM:QF muscle strength ratio (Table 1) for the subjects' involved and uninvolved leg and controls did not differ significantly as compared before 8-week HEP. The WOMAC score (Table 2) improved ( $\mathrm{p}<0.05$ ), and FTSTS time (Table 2) shortened $(\mathrm{p}<0.05)$ after 8 -week HEP in subjects, whereas these parameters remained lower $(\mathrm{p}<0.05)$ as compared with controls. 
Table 1. Mean $( \pm \mathrm{SE}$ ) values of knee active range of motion (aROM=deg), quadriceps femoris (QF) and hamstring (HM) muscle isometric strength (MVC) and HM:QF muscle strength ratio and gait parameters (stance time $(\mathrm{s})$, stride time $(\mathrm{s})$, step length $(\mathrm{m})$, velocity $(\mathrm{m} / \mathrm{s})$, and stride length $(\mathrm{m}))$ in subjects with knee osteoarthritis $(\mathrm{n}=10)$ before and after 8-weeks home exercise program (HEP) comparing the involved leg (IN) with the uninvolved leg (UN) and control subjects $(n=10)$.

\begin{tabular}{lccccc}
\hline \multirow{2}{*}{ Parameter } & \multicolumn{2}{c}{ Before } & \multicolumn{2}{c}{ After } & \multirow{2}{*}{ Controls } \\
\cline { 2 - 5 } & IN & UN & IN & UN & \\
\hline aROM & $108.3 \pm 3.4^{\text {ad }}$ & $84.9 \pm 7.4^{\mathrm{a}}$ & $115.0 \pm 2.4^{\mathrm{bcd}}$ & $96.6 \pm 5.7^{\mathrm{c}}$ & $126.0 \pm 1.9$ \\
\hline QF MVC & $0.11 \pm 0.02^{\mathrm{ad}}$ & $0.17 \pm 0.02^{\mathrm{a}}$ & $0.18 \pm 0.15^{\mathrm{b}}$ & $0.22 \pm 0.02^{\mathrm{b}}$ & $0.3 \pm 0.03$ \\
\hline HM MVC & $0.08 \pm 0.01^{\mathrm{ad}}$ & $0.12 \pm 0.01^{\mathrm{a}}$ & $0.12 \pm 0.02^{\mathrm{a}}$ & $0.15 \pm 0.02^{\mathrm{a}}$ & $0.24 \pm 0.01$ \\
\hline HM/QF MVC & $0.9 \pm 0.15$ & $0.75 \pm 0.07$ & $0.74 \pm 0.1$ & $0.70 \pm 0.008$ & $0.83 \pm 0.0$ \\
\hline Stance time & $0.69 \pm 0.02^{\mathrm{a}}$ & $0.71 \pm 0.02^{\mathrm{a}}$ & $0.73 \pm 0.09^{\mathrm{bc}}$ & $0.84 \pm 0.15$ & $0.55 \pm 0.01$ \\
\hline Stride time & $1.16 \pm 0.03^{\mathrm{a}}$ & $1.18 \pm 0.03^{\mathrm{a}}$ & $1.23 \pm 0.14$ & $1.28 \pm 0.16^{\mathrm{c}}$ & $0.96 \pm 0.02$ \\
\hline Step length & $0.71 \pm 0.04^{\mathrm{a}}$ & $0.71 \pm 0.03^{\mathrm{a}}$ & $0.66 \pm 0.05^{\mathrm{bc}}$ & $0.74 \pm 0.07$ & $0.85 \pm 0.03$ \\
\hline Velocity & $1.19 \pm 0.07^{\mathrm{a}}$ & $1.21 \pm 0.07^{\mathrm{a}}$ & $1.20 \pm 0.15^{\mathrm{c}}$ & $1.17 \pm 0.15^{\mathrm{c}}$ & $1.75 \pm 0.05$ \\
\hline Stride length & $1.17 \pm 0.05^{\mathrm{a}}$ & $1.21 \pm 0.04^{\mathrm{a}}$ & $1.23 \pm 0.1^{\mathrm{b}}$ & $1.15 \pm 0.06^{\mathrm{c}}$ & $1.41 \pm 0.03$ \\
\hline
\end{tabular}

${ }^{a}$ significant difference $(p<0.05)$ compared with the controls before 8-week HEP,

${ }^{b}$ significant difference $(p<0.05)$ compared with the pre-treatment level,

' significant difference $(p<0.05)$ compared with the controls after 8-week HEP,

${ }^{d}$ significant difference $(p<0.05)$ compared invloved leg with uninvolved leg

Table 2. Mean ( \pm SE) values of Five-Time-Sit-to-Stand (FTSTS) test time ( $\mathrm{s}$ ) and Western Ontario and McMaster Universities Osteoarthritis Index (WOMAC) score (points) in subjects with knee osteoarthritis $(n=10)$ before and after 8-weeks home exercise program (HEP) comparing results before and after 8-week HEP and control subjects $(n=10)$.

\begin{tabular}{lccc}
\hline Parameters & Before & After & Controls \\
\hline FTSTS & $26.3 \pm 6.8^{\mathrm{a}}$ & $16.02 \pm 2.5^{\mathrm{bc}}$ & $7.68 \pm 0.33$ \\
\hline WOMAC & $51.5 \pm 8.8^{\mathrm{a}}$ & $66.98 \pm 8.1^{\mathrm{bc}}$ & $99.3 \pm 0.54$ \\
\hline
\end{tabular}

${ }^{a}$ significant difference $(p<0.05)$ compared with the controls before 8-week HEP,

${ }^{b}$ significant difference $(p<0.05)$ compared with the pre-treatment level,

csignificant difference $(p<0.05)$ compared with the controls after 8-week HEP,

${ }^{d}$ significant difference $(p<0.05)$ compared involved leg with uninvolved leg.

After 8-week HEP, the stride length for the involved leg increased $(\mathrm{p}<0.05)$, whereas the stride length for the uninvolved leg was shorter $(\mathrm{p}<0.05)$ as compared with controls. The stance time was prolonged $(\mathrm{p}<0.05)$ and step length decreased $(\mathrm{p}<0.05)$ for the involved leg. The stance time, step length and 
velocity for the involved leg differed significantly $(\mathrm{p}<0.05)$ as compared with controls. Step length and stance time for the uninvolved leg and stride time for the involved leg did not differ significantly as compared with controls.

\section{DISCUSSION}

The main findings after 8-week HEP as compared with the pre-HEP level: (1) the QF muscle MVC force was increased for the involved and uninvolved leg and did not differ with controls; (2) sit-to-stand performance improved and disease-specific limitations were decreased (WOMAC).

This study demonstrated that in patients with knee OA, the QF muscle MVC force increased after 8-week HEP 39\% for the involved and 23\% for the uninvolved leg, and the HM muscle MVC force $33 \%$ and $20 \%$, respectively.

The HM:QF muscle strength ratio did not differ significantly between involved and uninvolved leg in subjects with knee OA and controls, whereas before 8-week HEP in subjects with knee OA the involved leg's HM muscle MVC force was higher than QF muscle MVC strength in three subjects, and after 8-week HEP in one subject. The study showed that increase bilateral QF muscle MVC force is possible with 8-week HEP. Participants in the study of McKay et al. [18] exercised 3 times per week for 6 weeks under supervision before TKA (circuit of bilateral lower body exercises) and achieved clinically significant increase in QF muscle strength, walking speed and mental health. Chun et al. [7] concluded that muscle strengthening in a treatment program is important for patients with severe knee OA. Baker et al. [3] found in their study that high-intensity, home-based strength training is effective in patients with knee pain and radiographic evidence of knee OA. Affected extremity strength deficit emerges by adaptive functional habits when knee pain and dysfunction affects subjects with knee OA for a long time before TKA (reduced physical activity).

During 8 weeks, HEP group subjects had an average 58 sessions, 30 minutes each. Subjects did HEP every day and exercised with both legs. They were physically active 58 minutes per day (walking, walking on stairs, gardening). During the first week, the subjects had problems with rise from chair and single leg stand. Since the third week the subjects described less pain in the knee joint during and after exercising; they were advised to increase the number of repetitions. Four weeks after starting they changed the easier Thera-band with a stronger one. The subjects who cancelled TKA (four out of 10) told that the involved leg does not disturb everyday life.

Esser and Bailey [11] concluded that exercising can be a powerful medicine for patients with knee OA. Carvalho et al. [5] found that home 
exercising (printed manual) with monthly reassessment by physiotherapist allowed in patients with knee OA to achieve a significant improvement in pain, knee ROM during flexion, muscle strength and functional capacity.

In our study, subjects with knee OA walked with altered gait. Difference between the involved leg and controls of the stride time, stance time and step length increased, whereas the velocity and stride length neared to the controls after 8-week HEP. Better indices of knee aROM during flexion and QF muscle MVC force are important factors for gait improvement. Esrafilian et al. [10] found that in patients with knee OA the stride length was significantly lower. Rutherford et al. [27] showed that the measured gait parameters worsened with disease progression. Shrader et al. [28] found that knee pain relief is adequate to improve gait in patients with painful medial compartment osteoarthritis of the knee after intra-articular injection.

In our study, the WOMAC score in subjects with knee OA increased $23 \%$ after 8-week HEP, whereas WOMAC score of controls stay 33\% higher. Rooks et al. [25] did not observe changes in the WOMAC total score. In their study, the exercise group performed water- and land-based exercises 3 times weekly over a 6-week period immediately prior to surgery. Similar tendencies in the WOMAC total score were found by McKay et al. [18] in their 6-week pre-rehabilitation exercise program conducted 3 times per week.

In our study, the FTSTS test time shortened $39 \%$ in subjects with knee OA after 8-week HEP, whereas controls performed this test 52\% quicker than HEP group. The similar results were established by Sled et al. [30] in their study after 8-week HEP of strengthening hip abductors in patients with medial knee OA. The study [6] showed that the FTSTS time was significantly longer in the end stage knee OA than in healthy controls.

The involved knee aROM during flexion improved after 8- week HEP $12 \%$, whereas the uninvolved knee improvement was $6 \%$. When comparing the involved and uninvolved leg with controls 8 weeks after HEP, the aROM difference was 23 and $9 \%$, respectively. Osteoarthritis is associated with changes in synovium, menisci, ligaments and muscles, and it is not realistic to expect full improvement in knee aROM after 8-week HEP. The maximal involved knee flexion was from 95 to $142^{\circ}$ in the study of Mizner et al. [19] before knee TKA, in the study of Otsuki et al. [21] the knee ROM before TKA was $117^{\circ}$, and in healthy controls $155^{\circ}$.

The present study has a limitation, the number of subjects with knee OA and healthy control subjects measured was small. Despite this, study indicates that in subjects with knee OA, the QF muscle MVC force, WOMAC score and knee aROM during flexion increased for the involved leg as compared to the uninvolved leg, and FTSTS test time shortened. 
This study demonstrated that in patients with knee OA was possible increase with 8-week HEP thigh muscle strength, knee aROM and strides length during gait for the involved leg, and improves sit to stand performance before TKA. Also the subjective condition that assessed with WOMAC score improved.

\section{ACKNOWLEDGEMENT}

We acknowledge that this study was supported by the Estonian Ministry of Education and Research, project No SF0180030s07, Estonian Science Foundation project No 7939, Estonian Ministry of Education and Research, Institutional research founding IUT20-58 and by European Union MYOAGE project, FP7 223576.

\section{REFERENCES}

1. Ackerman HN, Bennell KL. (2004) Does pre-operative physiotherapy improve outcome from lower limb joint replacement surgery? A systematic review. Aust J Physiother, 50: 25-30

2. Angst F, Ewert T, Lehmann S, Aeschilimann A, Stucki G. (2005) The factor subdimensions of the Western Ontario and McMaster Universities Osteoarthritis Index (WOMAC) help to specify hip and knee osteoarthritis. A prospective evaluation and validation study. J Rheumatol, 32: 1324-1330.

3. Baker KR, Nelson ME, Layne JE, Sarno R, Roubenoff R. (2001) The efficacy of home based progressive strength training in older adults with knee osteoarthritis: a randomized controlled trial. J Rheumatol, 28: 1655-1665.

4. Brown K, Topp R, Brosky JA, Lajoie AS. (2012) Prerehabilitation and quality of life three months after total knee atrhroplasty: a pilot study. Percept Mot Skills, 115: 765-774.

5. Carvalho NA, Bittar ST, Pinto FR, Ferreira M, Sitta RR. (2010) Manual for guided home exercises for osteoarthritis of the knee. Clinics, 65: 775-780.

6. Christiansen CL, Stevens-Lapsley JE. (2010) Weight-bearing asymmetry in relation to measures of imparement and functional mobility for people with knee OA. Arch Phys Med Rehabil, 91: 1524-1528.

7. Chun S-W, Kim K-E, Jang S-N, Kim KI, Paik NJ, Kim KW, Jang HC, Lim JY. (2013) Muscle strength is the main associated factor of physical performance in older adults with knee osteoarthritis regardless of radiographic severity. Arch Gerontol Geriatr, 56: 377-382.

8. Csuka M, McCarty DJ. (1985) Simple method for measurement of lower extremity muscle strength. Am J Med, 78: 77-81.

9. Davis RB, Ounpuu S, Tyburski DS, Gage JR. (1991) A gait analysis collection and reduction technique. Hum Mov Sci, 10: 575-587. 
10. Esrafilian A, Karimi MT, Amiri P, Fatoye F. (2013) Performance of subjects with knee osteoarthritis during walking: differential parameters. Rheumatol Int, 33: 1753-1761.

11. Esser S, Bailey A. (2011) Effects of exercise and physical activity on knee osteoarthritis. Curr Pain Headache Rep, 15: 423-430.

12. Gogia PP, Braatz JH, Rose SJ, Norton BJ. (1987) Reliability and validity of goniometric measurements at the knee. Phys Ther, 67: 192-195.

13. Harding GT, Hubley-Kozey CL, Dunbar MJ, Stanish WD, Wilson JL. (2012) Body mass index affects knee joint mechanics during gait differently with and without moderate knee osteoarthritis. Osteoarthritis Cartilage, 20: 1234-1242.

14. Hurley MV, Scott DI. (1998) Improvements in quadriceps sensiomotor function and disability of patients with knee osteoarthritis following a clinically practicable exercise regime. Br J Rheumatol, 37: 1181-1187.

15. Jaggers JR, Simpson CD, Frost KL, Quesada PM, Topp RV, Swank AM, Nyland JA. (2007) Prerehabilitation before knee arthroplasty increases postsurgical function: a case study. J Strength Cond Res, 21: 632-634.

16. Kellegren JH, Lawrence JS. (1957) Radiological assessment of osteo-arthrosis. Ann Rheum Dis, 16: 494-502.

17. Lauretani F, Russo CR, Bandinelli S, Bartali B, Cavazzini C, Di Iorio A, Corsi AM, Rantanen T, Guralnik JM, Ferrucci L. (2003) Age-associated changes in skeletal muscles and their effect on mobility: an operational diagnosis of sarcopenia. J Appl Physiol, 95: 1851-1860.

18. McKay C, Prapavessis H, Doherty T. (2012) The effect of a prerehabilitation exercise program on quadriceps strength for patients undergoing total knee arhroplasty: a randomized controlled pilot study. PM R, 4: 647-656.

19. Mizner RL, Petterson SC, Snyder-Mackler L. (2005) Quadriceps streength and the time course of functional recovery after total knee arthroplasty. J Orthop Sports Phys Ther, 35: 424-436.

20. Odding E, Valkenburg HA, Algra D, Vandenouweland FA, Grobbee DE, Hoffman A. (1996) The association of abnormalities on physical examination of the hip and knee with locomotor disability in the Rotterdam study. Br J Rheumatol, 35: 884-890.

21. Otsuki T, Nawata K, Okuno M. (1999) Quantitative evaluation of gait pattern in patients with osteoarthrosis of the knee before and after knee total arthroplasty. Gait analysis using a pressure measuring system. J Orthop Sci, 4: 99-105.

22. Philadelphia Panel. (2001) Philadelphia Panel evidence-based clinical practice guidelines on selected rehabilitation interventions for knee pain. Phys Ther, 81: 1675-1700.

23. Ramzjou H, Finkelstein JA, Yee A, Holtby R, Vidmar M, Ford M. (2009) Relationship between preoperative patients characteristics and expectations in candidates for total knee arthroplasty. Physiother Can, 61: 38-45.

24. Rodgers JA, Garvin Kl, Walker CW, Morford D, Urban J, Bedard J. (1998) Preoperative physical therapy in primary total knee arthroplasty. J Arthroplasty, 13: $414-421$. 
25. Rooks DS, Hunag J, Bierbaum BE, Bolus SA, Rubano J, Connolly CE, Alpert S, Iversen MD, Katz JN. (2006) Effect of preoperative exercise on measures of functional status in men and women undergoing total hip and knee arthroplasty. Arthritis Rheum, 55: 700-708.

26. Roos EM, Juhl CB. (2012) Osteoarthritis 2012 year in revew: rehabilitation and outcomes. Osteoarthritis Cartilage, 20: 1477-1483.

27. Rutherford DJ, Hubley-Kozey CL, Stanish WD, Dubnar MJ. (2011) Neuromuscular alterations exist with knee osteoarthritis presence and severity despite walking velocity similarities. Clin Biomech, 26: 377-383.

28. Shrader MW, Draganich LF, Pottenger LA, Piotrowski GA. (2004) Effects of knee relief in osteoarthritis on gait and stair-stepping. Clin Orthop Relat Res, 412: $188-193$.

29. Skou ST, Roos EM, Laursen MB, Rathleff MS, Arendt-Nielsen L, Simonsen $\mathrm{OH}$, Rasmussen S. (2012) Total knee arthroplasty plus physical and medical therapy or treatment with physical and medical therapy alone: a randomised controlled trial in patients with knee osteoarthritis (the MEDIC-study). BMC Musculoskelet Disord, 13: 67.

30. Sled EA, Khoja L, Deluzio KJ, Olney SJ, Culham EG. (2010) Effect of a home program of hip abductors exercises on knee joint loading, strength, function, and pain in people with knee osteoarthritis: a clinical trial. Phys Ther, 90: 895-904.

31. Stark T, Walker B, Philips JK, Fejer R, Beck R. (2011) Hand-held dynamometry correlation with the gold standard isokinetic dynamometry: a systematic review. PM R, 3: 472-479.

32. Steultjens MP, Dekker J, van Baar ME, Oostendrop RA, Bijlsma JW. (2000) Range of joint motion and disability in patients with osteoarthritis of the knee or hip. Rheumatol, 39: 955-961.

33. Sutherland DH. (2002) The evolution of clinical gait analysis. Part II Kinematics. Gait Posture, 16: 159-179.

34. Yoshimura N, Muraki S, Oka H, Tanaka S, Kawaguchi H, Nakamura K, Akune T. (2012) Accumulation of metabolic risk factors such as overweight, hypertension, dyslipidaemia, and impaired glucose tolerance raises the risk of occurrence and progression of knee osteoarthritis: a 3-year follow-up of the ROAD study. Osteoarthristis Cartilage, 20: 1217-1226.

\section{Correspondence to:}

Jelena Sokk

Institute of Sport Sciences and Physiotherapy,

University of Tartu, Jakobi 5, 51014, Tartu, Estonia

Tel./Fax: +372 7376286

E-mail: jelena.sokk@ut.ee 